\title{
DiZIONARIO DI COLLOCAZIONI O DIZIONARIO DI COMBINAZIONI? UNA PROSPETTIVA USER-ORIENTED
}

\section{Dicionário de colocações ou dicionário de combinações? Uma perspectiva user-oriented}

\section{Word Collocation Dictionary or Word Combination Dictionary? A User-oriented Perspective}

Francesco Urzi ${ }^{*}$

\begin{abstract}
Il presente contributo prende in esame alcune delle scelte metodologiche che il lessicografo che intenda a realizzare un dizionario di collocazioni si trova ad affrontare. Il lemmario deve coincidere con un determinato strato del lessico (ad es. il "vocabolario di base") oppure essere il risultato di un procedimento bottom-up che ne faccia la "proiezione" delle collocazioni effettivamente raccolte (ad es. mediante estrazione da corpora)? Considerato che le collocazioni, secondo il grado più o meno elevato di coesione e di rigidità sintattica esibite, si distribuiscono lungo un continuum delimitato da un lato dalle combinazioni libere e dall'altro dalle espressioni idiomatiche, come trattare i casi-limite? Ad esempio, che «collocazione» trovare nel dizionario per le unità polirematiche? Più in generale, quanto teorico e quanto orientato all'utente deve essere un dizionario di collocazioni? Infine, nei lemmi nominali polisemici, l'ordinamento semantico della voce deve ricalcare quello dei dizionari generali o occorre in determinati casi applicare una sorta di "lente di ingrandimento" che promuova al rango di accezioni quei sensi cui corrispondano collocati verbali specifici?
\end{abstract}

PAROLE CHIAVE: Collocazioni; Combinazioni libere e lessicali; Unità polirematiche; Idiomatismi; Ordinamento semantico.

RESUMO: O presente artigo analisa algumas das escolhas metodológicas a serem afrontadas por um lexicógrafo que pretenda elaborar um dicionário de colocações. O conjunto de entradas (nomenclatura) deve coincidir com um determinado nível do

*Parlamento Europeo

combinazioni.lessicali@gmail.com (ORCID:0000-0001-7013-8441)

DOI: http://dx.doi.org/10.11606/issn.2238-8281.v0i40p114-126 
léxico (por exemplo, o "vocabulário de base")? Ou deve ser o resultado de um procedimento bottom-up que faça a "projeção" de colocações efetivamente coletadas (por exemplo, mediante uso de corpora)? Considerando que as colocações, segundo seu grau de coesão e de rigidez sintática mais ou menos elevado, distribuem-se ao longo de um continuum delimitado, de um lado, pelas combinações livres e, de outro, pelas expressões idiomáticas, como tratar os casos-limite? Por exemplo, que colocação encontrar no dicionário para as unità polirematiche? Em termos gerais, em que medida deve ser "teórico" ou "orientado ao usuário" um dicionário de colocações? Enfim, nas entradas nominais polissêmicas, a ordem semântica do verbete deve seguir aquela dos dicionários gerais ou, em determinados casos, é necessário aplicar uma espécie de "lente de aumento" que promova à posição de acepção aqueles sentidos que selecionam colocados verbais específicos?

PALAVRAS CHAVE: Colocações; Combinações livres e lexicais; Unità polirematiche; Idiomatismos; Ordem semântica.

ABSTRACT: This paper examines some of the methodological choices faced by the lexicographer wishing to compile a dictionary of collocations. Should entries be exclusively come from a 'basic vocabulary' or should their list be the result of a 'bottom-up' process, i.e. an ex-post extraction of the bases of all collocations collected (e.g. by means of extraction from corpora)? In terms of cohesion and syntactic rigidity, collocations distribute themselves along a continuиm whose boundaries are delimited by free combinations on the one side and by idioms on the other. Which 'borderline' word combinations, such as multi-word units, should be included in the dictionary? In more general terms, how theoretical and how user-oriented should a collocations dictionary be? Finally, should sense ordering in polysemous noun entries reflect that of general dictionaries or should a sort of 'magnifying glass' be applied, in order to capture those sense variations which select specific verb collocations?

KEYWORDS: Collocations; Free and word combinations; Multi-word units; Idioms; Sense ordering. 


\section{Introduzione}

Le collocations (da cui il termine italiano 'collocazioni') sono definite dall' Oxford English Dictionary come "a pair or group of words that frequently occur together". Simone (2013, p. 260) dà per le collocazioni la seguente definizione: "sequenze di parole che ricorrono in combinazioni stabili sull'asse sintagmatico" [corsivo mio], dove la nozione di stabilità richiama, più che la frequenza, la coesione tra i costituenti. La presente indagine si propone di esaminare $\mathrm{i}$ principali problemi connessi al trattamento lessicografico delle combinazioni di parole che per la loro frequente co-occorrenza o minore o maggiore coesione lessicale e sintattica ruotano attorno alla nozione di collocazione.

In particolare l'autore intende proporre nel presente articolo alcune delle riflessioni sottese alla redazione del DCL - Dizionario delle Combinazioni Lessicali (URZİ, 2009), il cui scopo, come la scelta stessa del titolo dimostra, è di mettere a disposizione uno strumento di consultazione che sfrutti l'asse sintagmatico per la ricerca di un elemento lessicale. Testimonia l'interesse degli studiosi per questo aspetto della fraseologia il numero dei dizionari combinatori pubblicati in rapida successione per l'italiano dopo il DCL: Russo, Domenico (2010), MdD. Modi di Dire. Lessico Italiano delle Collocazioni, Aracne, Roma; Lo Cascio, Vincenzo (2012), Dizionario Combinatorio Compatto Italiano, John Benjamins, Amsterdam; Tiberii, Paola (2012), Dizionario delle collocazioni, Zanichelli, Bologna; Lo Cascio, Vincenzo (2013), Dizionario Combinatorio Italiano, John Benjamins, Amsterdam. Significativa anche la pubblicazione nello stesso periodo del Grande Dizionario Analogico della lingua italiana di Raffaele Simone (SIMONE, 2010).

\section{Dalle combinazioni libere alle espressioni idiomatiche}

Il mero criterio della co-occorrenza statistica non basta a qualificare una combinazione come collocazione. Ad esempio, in cercare le chiavi, 'cercare' è sì un verbo tipicamente associato a 'chiave', ma lo è sul piano della congruenza concettuale; in altre parole è nella natura stessa delle chiavi, in quanto oggetto fisico di piccole dimensioni, il poter essere perduto, cercato o trovato. In questo caso si parla di combinazioni libere.

Sono invece considerate collocazioni a pieno titolo espressioni come colmare un divario, dove il nome può essere combinato solo con un numero limitato di verbi (ad es. 'colmare', ma non '*riempire'). In casi come questo un apprendente straniero avrebbe difficoltà a reperire il verbo corretto mediante la semplice traduzione del verbo equivalente.

La collocazione può presentare un certo grado di rigidità sintattica, come in sporgere denuncia, dove tra 'sporgere' e 'denuncia' è possibile interporre solo una o due parole (ha sporto subito denuncia). Si parla in questi casi di collocazioni ristrette. Vi sono anche casi di monocollocabilità (come in nodo scorsoio) in cui la combinazione mostra una rigidità sintattica assoluta, che non ammette alcuna interposizione. ${ }^{1}$ Quando l'espressione perde la sua composizionalità, ossia quando il suo significato non è più derivabile da quello dei suoi componenti, si

1 Esula dallo scopo del presente studio una descrizione «tassonomica» delle collocazioni in funzione del maggiore o minor grado di restrizione lessicale e sintattica tra i costituenti. 
entra nel dominio delle espressioni idiomatiche. È idiomatica ad esempio l'espressione vuotare il sacco, il cui significato, costruito su una similitudine, deve essere memorizzato dall'apprendente di L2 così com'è.

Le collocazioni, dunque, in funzione del grado più o meno elevato di coesione e di rigidità sintattica, si distribuiscono lungo un continuиm delimitato da un lato dalle combinazioni libere, dall'altro dalle espressioni idiomatiche. Hausmann (1985, pp. 118-129) le ha opportunamente definite "prodotti semilavorati della lingua" (Halbfertigprodukte der Sprache).

\subsection{Dalla base al collocato}

Nella loro forma più caratteristica le collocazioni mostrano una struttura binaria: una base e un collocato (JEŽEK, 2011). La base della collocazione può essere considerata come il "portale di accesso" dal costituente noto a quello non noto (o momentaneamente non disponibile nel lessico mentale dell'utente al momento della produzione linguistica); è la categoria lessicale dalla quale viene avviato il processo di generazione dell'espressione ed è anche quella che viene di solito posta a lemma nei dizionari combinatori.

I tipi di collocazioni di maggiore rilevanza lessicografica sono illustrati dagli esempi seguenti (la base è in maiuscolo, il collocato in corsivo):

$\begin{array}{ll}\text { Verbo + Nome: } & \text { acquistare la CITTADINANZA italiana } \\ \text { Nome + Verbo: } & \text { il DESTINO si è accanito contro di lui } \\ \text { Agg. + Nome: } & \text { comportarsi da perfetto GENTILUOMO } \\ \text { Nome + Agg.: } & \text { una persona di una GENTILEZZA squisita } \\ \text { Nome + Nome: } & \text { un atto di INDISCIPLINA/un ventaglio di POSSIBILITÀ } \\ \text { Verbo + Avv.: } & \text { il mare MORMORAVA sommessamente } \\ \text { Agg. + Avv.: } & \text { prezzi fortemente COMPETITIVI }\end{array}$

Il percorso di ricerca base $\Rightarrow$ collocato risponde di volta in volta a precise esigenze di produzione linguistica (in parentesi negli esempi in appresso):

Nome $\Rightarrow$ Verbo

Che cosa si fa tipicamente della/con la CITTADINANZA?

Cosa può fare il DESTINO a una persona?

Nome $\Rightarrow$ Agg

Come qualificare un GENTILUOMO per accentuarne il tratto di signorilità?

Come qualificare la GENTILEZZA di una persona?

Nome $\Rightarrow$ Nome

Come denotare una singola manifestazione di INDISCIPLINA o un certo numero di POSSIBILITÀ? 
Verbo $\Rightarrow$ Avv.

Come modificare il senso di MORMORARE per indicare il rumore leggero dello

sciabordio delle onde?

Agg. $\Rightarrow$ Avv.

Come intensificare l'aggettivo COMPETITIVO con riferimento ai prezzi?

Tale percorso di ricerca presuppone una strutturazione del dizionario combinatorio che metta a lemma le basi (negli esempi di cui sopra: Nome, Verbo o Aggettivo). L'ordine di ricerca inverso, dal collocato alla base, non appare tale da soddisfare le normali esigenze della produzione linguistica, ma è oggi comunque reso possibile dai link testuali forniti dai dizionari elettronici.

\section{La rappresentazione delle collocazioni in un dizionario generale}

I dizionari generali propongono di solito le collocazioni come esempi tipici d'uso nell'ambito di questa o quella accezione. Il Conciso Treccani (SIMONE, 1998) è stato il primo a includere le collocazioni in una specifica sezione Espressioni posta in fondo alla voce. Lo Zingarelli (2016) le tratta invece all'interno degli «Inserti di nomenclatura», dove ad es. s.v. Abito troviamo le caratteristiche e le azioni tipicamente associate al lemma (aggettivi: borghese, militare, ecclesiastico [...]; verbi: indossare, infilare, portare [...]), secondo l'impostazione tipica di un dizionario analogico. Questi inserti arricchiscono senza dubbio il dizionario, ma non lo rendono lo strumento di consultazione ideale per la ricerca di collocazioni. Gli inserti sono infatti disponibili per un numero ristretto di voci (per la precisione 118) e l'assenza di un criterio preciso e "preventivamente noto" che ci dica quali lemmi dispongano di tale corredo lessicale induce l'utente a dimenticare che il dizionario offre questa risorsa aggiuntiva.

A questo inconveniente si potrebbe ovviare distribuendo le collocazioni fra le varie accezioni della base nominale. Ma anche questo procedimento non è scevro di conseguenze a livello redazionale e comporta probabilmente, come vedremo al paragrafo 5, un ripensamento della struttura interna degli articoli del dizionario e dell'ordinamento semantico delle accezioni. 


\section{La rappresentazione delle collocazioni nel dizionario combinatorio}

\subsection{Scelta dei lemmi}

Nella costruzione del lemmario di un dizionario combinatorio (le "basi" delle collocazioni) è forte la tentazione di limitarsi ai circa 6700 lemmi che compongono il vocabolario di base del Gradit (DE MAURO, 2007) nell'intento di rappresentare nel dizionario le collocazioni più frequenti e utilizzate ${ }^{2}$. Se tuttavia si considerano le collocazioni come entità lessicali a sé stanti, il criterio dell'appartenenza dei costituenti singolarmente considerati a questo o quello strato del lessico diviene in molti casi irrilevante. Vediamo due esempi.

Nella collocazione cadere nell'oblio - collocazione "ristretta" in quanto non vi sono altri possibili candidati alla posizione di verbo - la base nominale 'oblio' appartiene al vocabolario "comune", ossia alle ca. 40.000 parole note "a chiunque abbia un livello medio-superiore di istruzione" (DE MAURO, 1999-2003) e il collocato verbale 'cadere' al vocabolario fondamentale (ossia i ca. 2.000 vocaboli più frequenti in assoluto). L'appartenenza della base lessicale ('oblio') a una fascia lessicale diversa da quella di base non appare tuttavia motivo sufficiente per non trattare la collocazione. In altre parole, il fatto che all'utente possa essere nota una base appartenente a una fascia lessicale superiore non autorizza a presumere che l'utente stesso (magari un apprendente di L2) sia in grado di produrre il collocato solo perché questo appartiene a una fascia lessicale inferiore. Analogamente, nella collocazione esercitare un arbitrato (ad esempio nella frase l'arbitrato è stato esercitato conformemente agli standard delle Nazioni Unite) la base nominale è costituita da un 'termine specialistico' (marca TS nel Gradit) cui si associa un verbo (esercitare) appartenente al lessico fondamentale. Eppure l'associazione tra 'esercitare' e 'arbitrato' è tutt'altro che ovvia nella produzione di un testo. Per tali motivi il processo di formazione del lemmario in un dizionario combinatorio dovrebbe seguire un procedimento bottom-up il cui risultato sia la "proiezione" delle basi delle collocazioni raccolte (ad es. mediante estrazione da corpora) per la stesura del dizionario.

Lo status delle collocazioni come entità lessicali a sé stanti assume rilievo anche al momento di stabilire se lemmatizzare determinati sintagmi lessicalizzati (quali le unità polirematiche ${ }^{3}$, che saranno trattate più diffusamente al paragrafo 4.4), trattandoli come basi a pieno titolo. In intentare un'azione penale il verbo è un collocato specifico del SN azione penale e non semplicemente di 'azione'.

2 Ad esempio, in Häcki Buhofer et al. (2014, p. XIX) le combinazioni riportate sono state estratte da un corpus ad hoc, partendo da un Basisworschatz di ca. 2000 parole. Ciò fa sì che una collocazione come eine Allianz schmieden non venga registrata nel dizionario per il solo fatto che la base nominale 'Allianz' non appartiene al vocabolario di base preso in considerazione.

3 Termine assai in voga in Italia per designare i sintagmi lessicalizzati composizionali. 


\subsection{Collocazioni multiparola}

I tipi di collocazioni elencati al par. 2.1 ci mostrano una struttura binaria a due costituenti, la base e il collocato. Consideriamo ora le due collocazioni seguenti:

1) Prendere una boccata d'aria, con struttura V+SN e con una base (b. d'aria) che è a sua volta una collocazione $\mathrm{N}+\mathrm{N}$.

2) [La proposta] ha ricevuto favorevole accoglienza, con struttura $\mathrm{V}+\mathrm{N}$ e con una base che è a sua volta una collocazione $\mathrm{A}+\mathrm{SN}(f$. accoglienza $)$.

Lo schema base/collocato sopra illustrato resta valido, ma le basi nominali sono costituite rispettivamente dai sintagmi boccata d'aria e favorevole accoglienza. Se nel primo caso può esservi sinonimia di significato tra prendere aria e prendere una boccata d'aria, nel secondo la presenza di favorevole è funzionale alla formazione della collocazione $\mathrm{V}+\mathrm{SN}$ giacché non avrebbe senso affermare semplicemente che una proposta *ha ricevuto accoglienza. Sul piano lessicografico il problema consiste nel segnalare, magari con opportuni mezzi grafici, che in determinati casi è indispensabile la presenza di un terzo elemento lessicale. Un'altra e più radicale soluzione consisterebbe nel rendere boccata d'aria e favorevole accoglienza lemmi a pieno titolo, esattamente come per la già vista azione penale. Tuttavia questo procedimento finirebbe forse per rendere più difficoltosa la consultazione, dal momento che la cultura lessicografica (anche di coloro che i dizionari solo consultano) è ancora saldamente ancorata all'approccio monorematico.

Il numero dei costituenti della collocazione può però essere ancora superiore. Vediamo due esempi:

1) In un vecchio adagio sostiene che chi cambia non è felice il collocato verbale (sostenere) può essere associato alla base 'adagio' solo aggiungendo un'intera frase oggettiva (che chi cambia non è felice o altro antico motto proverbiale) che saturi la valenza del verbo.

2) Nella sequenza (invero stereotipa, ma assai frequente nella prosa giornalistica) asfalto reso viscido dalla pioggia la base 'asfalto' ha come collocato il SA reso viscido dalla pioggia. Chi volesse ricercare questa espressione in un dizionario analogico partendo da 'asfalto' troverebbe sicuramente 'viscido' o 'scivoloso', ma nulla che indichi che la scivolosità è diretta conseguenza del bagnato; se per contro la ricerca viene avviata a partire dalla base nominale 'pioggia' difficilmente si troverà il sintagma verbale rendere viscido. Il SA complesso reso viscido dalla pioggia può pertanto ben figurare tra i collocati aggettivali in quanto specificamente riferibile a uno scenario situazionale, a un frame del tipo guida su strada bagnata e, come si vedrà al paragrafo 5 , è proprio la maggiore granularità semantica della voce di un dizionario combinatorio quella che permette il più agevole reperimento di un collocato adatto ${ }^{4}$.

4 Per fare un esempio di trattamento lessicografico, lo status di sintagma del collocato aggettivale viene segnalato solo indirettamente nel DCL, che registra l'espressione dando diverso rilievo grafico ai costituenti (asfalto reso viscido dalla pioggia), in ossequio alla tradizione monorematica della lessicografia italiana. 


\subsection{Inclusione di combinazioni libere e di espressioni semi-idiomatiche}

Quando l'espressione si avvicina ai limiti del continuum combinazioni libere-idiomatismi, un approccio lessicografico inclusivo ${ }^{5}$ sembra essere la scelta lessicografica più appropriata. Per esempio, l'espressione riposare sugli allori ha certamente carattere idiomatico, ma conserva un certo grado di composizionalità, visto che 'alloro' può essere usato in altri contesti nel senso di 'vittoria, trionfo' (ad es. in conquistare l'alloro olimpico). La scelta di includere in un dizionario combinatorio riposare sugli allori offre così una soluzione linguistica a chi (per stanchezza, vuoti momentanei di memoria ecc.) pur disponendo della base 'alloro' nel proprio lessico mentale, abbia difficoltà a reperire nel paradigma (dormire, riposare, *giacere) il verbo che correttamente completa l'espressione.

Lo stesso approccio inclusivo sembra opportuno anche sul versante opposto delle combinazioni libere. L'utente vede nel dizionario di collocazioni uno strumento di consultazione combinatorio e non è realistico presumere che, prima di effettuare la ricerca, si fermi a riflettere per valutare lo status lessicologico dell'espressione ricercata, ossia se l'elemento lessicale ricercato forma con la base una vera collocazione o no. L'utente del dizionario potrebbe anche essere un apprendente straniero cui serva conoscere ad es. taluni verbi comuni combinabili con la base 'chiave', ad es. 'perdere' o 'smarrire', che pur non formando con 'chiave' una collocazione (si può perdere o smarrire qualunque oggetto), possono comunque risultare utili a completare il cloze mentale. In altre parole la decisione se registrare o meno una combinazione di parole (collocazione o combinazione più o meno libera) dipende in ultima analisi dalla risposta che il lessicografo dà alla domanda: può presentarsi un'esigenza di produzione linguistica in cui data una base (chiave) l'utente possa aver bisogno di reperire un secondo costituente (perdere, smarrire) indipendentemente dal fatto che la combinazione risultante formi o meno una collocazione?

Questo approccio largo (inserimento nel dizionario di talune combinazioni che non sono collocazioni stricto sensu), che pur viola la distinzione teorica tra combinazioni libere, collocazioni e idiomatismi, ricorda la prassi, tipica della lessicografia inglese, di elevare determinati nomi propri (ad esempio di luoghi e persone famosi) al rango di lemma ${ }^{6}$, violando la distinzione teorica fra lessico ed enciclopedia che contraddistingue invece la lessicografia italiana. L'approccio della lessicografia inglese ha preferito infatti pragmaticamente considerare i nomi propri come fatti di lingua a pieno titolo. Questo approccio teorico flessibile spiega anche perché tre dei dizionari combinatori citati nell'Introduzione non rechino nel titolo il termine 'collocazione'.

5 Per «approccio inclusivo» intendo la scelta di ricomprendere talune espressioni idiomatiche che si situano ai limiti del continuum collocazioni-idiomatismi, alla condizione sopra indicata, ossia la presenza di un certo grado di composizionalità che a sua volta permette all'utente di lanciare la ricerca a partire da una base (nell'esempio riportato, 'alloro').

6 Così fa ad esempio il New Oxford Dictionary of English (2001). 


\subsection{Trattamento delle polirematiche}

Altro dilemma che affligge gli autori di dizionari combinatori è quale trattamento riservare, nel corpo della voce, alle unità polirematiche, ossia se e quando esse vadano considerate combinazioni lessicali funzionalmente equiparabili alle collocazioni.

In una recente rassegna analitica dei recenti dizionari combinatori dell'italiano, Fesenmeyer considera la presenza di unità polirematiche nei dizionari di questo tipo un criterio per stabilire il «concetto di combinazione/collocazione applicato»e ritiene che:

il loro numero [il numero di polirematiche nei dizionari esaminati, N.d.A] indurrà comunque a ridimensionare un po' la significatività delle cifre relative alla quantità delle combinazioni/collocazioni ritenute. (FESENMEYER, 2015, pp. 110-131)

In altre parole, le polirematiche sarebbero da «portare in detrazione» dal numero totale di combinazioni/collocazioni presenti nel dizionario.

Masini così spiega la differenza fra collocazioni e polirematiche:

mentre le polirematiche sono coese sia dal punto di vista paradigmatico sia dal punto di vista sintagmatico, le collocazioni sono strutture fisse solo dal punto di vista paradigmatico (poiché, dato un certo tipo di base $X$, si avrà necessariamente un dato collocato Y), ma non sintagmatico (ovvero l'espressione può subire manipolazioni di tipo sintattico). Un'espressione come bandire un concorso sarà quindi una collocazione e non una polirematica, perché, pur essendo obbligatoria la scelta di bandire in collegamento con concorso, la costruzione può subire modificazioni sintattiche: ad es., si può avere la costruzione passiva (il concorso è stato bandito) o modificare un elemento interno (hanno bandito molti concorsi). (2011, p. 1111)

Tuttavia questo criterio non è assoluto, come dimostra l'espressione fare cilecca che, seppur definita polirematica da Voghera (2004, p. 59), supera solo il primo dei due test di Masini, quello dell'impossibilità della costruzione passiva (*è stata fatta cilecca), ma non il secondo, ossia quello dell'inammissibilità dell'interposizione di altre parole (si può dire infatti che il sistema di sicurezza ha fatto inaspettatamente cilecca). Bisetto (2004, p. 36) definisce le polirematiche come l'esito di un processo di lessicalizzazione che fa assumere al costrutto un significato non composizionale, citando come esempio tipico conferenza stampa, che non è un tipo di conferenza. Se ci si attiene rigidamente al criterio della non composizionalità come elemento distintivo delle polirematiche si è autorizzati a concludere che le polirematiche non dovrebbero trovare spazio in un dizionario di collocazioni, poiché diverrebbe impraticabile il percorso di ricerca base $\Rightarrow$ collocato, che implica la scomponibilità del sintagma. Tuttavia, De Mauro (2005, p. 89) introduce per le polirematiche una definizione più articolata, aggiungendo come criterio distintivo autonomo, oltre a quelli della non composizionalità e della più o meno forte cristallizzazione lessicale e sintattica, anche quello della "presenza significativa in linguaggi tecnico-specialistici”, adducendo un esempio tratto dalla fisica (particella elementare). 
In linea con le conclusioni raggiunte al paragrafo 4.3 a proposito del "diritto di cittadinanza" di determinate espressioni semi-idiomatiche in un dizionario di collocazioni, almeno per le espressioni che devono il loro status di polirematiche alla loro appartenenza a un linguaggio tecnico-specialistico si verificano tutte le condizioni per ipotizzare come produttivo il percorso di ricerca tipico che abbiamo visto per le collocazioni. È realistico ad esempio ipotizzare che, a partire dalla base 'porto', l'utente abbia l'esigenza di risalire a porto marittimo (e non a porto di mare, che ha una valenza idiomatica) perché il testo richiede l'uso di un termine politico-amministrativo. Si deve anzi presumere che le polirematiche con questa struttura (N+A di relazione) si prestino sovente a questo tipo di ricerca lessicale ${ }^{7}$.

\section{La strutturazione interna delle voci nel dizionario tradizionale e nel dizionario combinatorio}

Come si è detto al paragrafo 3 , il dizionario generale riporta sì un certo numero di collocazioni, ma solo come esempi d'uso. È anche noto che uno degli errori da non commettere nella compilazione di un dizionario generale è di strutturare le accezioni del lemma sulla base degli esempi (a volte letterari) che vi sono proposti. Come afferma Marello:

Bisogna evitare il pericolo di promuovere ad accezione quello che è il senso
specifico che una parola acquista in un enunciato. La lessicografia italiana, nata con
una tradizione di esempi d'autore, corre talvolta il rischio di subire il fascino del
bell'esempio; (...) [si può finire] per frammentare eccessivamente le proprie accezioni
inseguendo sensi specifici di un contesto, non riuscendo a dare un'immagine
complessiva dell'evoluzione semantica di una parola. (1996, p. 119)

Una volta promosse da esempi d'uso a oggetto specifico di trattazione, le collocazioni finiscono però per costruire uno schema di accezioni del lemma di base non esattamente coincidente con quello di un dizionario semasiologico. Vediamo alcuni esempi.

Nel Gradit (DE MAURO, 2007) l'accezione 1 della voce 'Dichiarazione' propone la seguente definizione:

1. Il dichiarare, il dichiararsi; il discorso o lo scritto con cui si dichiara: fare una $d$.; raccogliere le dichiarazioni dei testimoni; firmare, sottoscrivere una d.; rilasciare una d. ai giornalisti, chiedere di fare una d., dichiarazioni di stima, di amicizia.

Si vede come la definizione, pur coerente, riunisca in una stessa accezione la sfera privata (d. di stima e di amicizia) e la sfera pubblica (rilasciare una d. ai giornalisti). In un dizionario di collocazioni, tuttavia, le due sfumature di senso possono richiedere due collocati verbali differenti, come 'fare' (una d. d'amore) e 'rilasciare' (una d. ai giornalisti). In altre parole, la distinzione fra le due dimensioni, quella pubblica e quella privata, diviene rilevante per la

7 Tipicamente da parte di un apprendente di L2, ma non è difficile ipotizzare situazioni in cui anche un madrelingua possa avere analoghe esigenze. 
necessità di utilizzare due collocati verbali diversi. Se estendiamo l'analisi ad altre coppie di combinazioni $\mathrm{V}+\mathrm{N}$, come ad es.:

\author{
fare appello vs. rivolgere un appello \\ erogare servizi vs. prestare servizi \\ stendere un contratto vs. rogare un contratto \\ evadere un ordine vs. collocare un ordine (di borsa) \\ chiedere asilo vs. richiedere asilo
}

si vede come ciascuna delle due collocazioni della coppia faccia riferimento a un campo di interpretazione distinto, che influenza a sua volta la selezione del collocato verbale (ad es. i servizi sono erogati dallo Stato, ma sono prestati da professionisti). Un dizionario generale dell'uso che riporti sistematicamente le collocazioni distribuendole fra le varie accezioni dovrà allora rendere esplicito il frame che condiziona la scelta del collocato, mentre un dizionario combinatorio dovrà optare per una strutturazione più granulare delle accezioni, secondo uno schema desunto direttamente dalle collocazioni riportate nella voce.

\title{
6. Conclusioni
}

Una nozione di collocazione che funzioni sul piano lessicografico, ossia che permetta a chi usa il dizionario combinatorio di disporre di un'efficace porta di accesso orizzontale (sintagmatica) al materiale lessicale ricercato, presuppone una struttura binaria base + collocato.

In sede di scelta delle basi da mettere a lemma sarà opportuno prescindere dall'appartenenza delle basi a questo o a quello strato del lessico e costruire il lemmario secondo un procedimento ex post che parta dalle collocazioni effettivamente raccolte (ad es. mediante estrazione da corpora). Resta aperta la questione se e in che misura lemmatizzare anche determinati sintagmi lessicalizzati (tipicamente unità polirematiche) che hanno proprietà combinatorie parzialmente diverse da quelle della testa del sintagma (ad es. azione penale), specie se si tiene conto della tradizione monorematica della lessicografia italiana e della corrispondente «cultura della consultazione» da parte degli utenti.

Le collocazioni trattate potranno avere come collocato un sintagma più o meno complesso quando la sola testa del sintagma non basta a formare una collocazione ad esempio perché si fa riferimento a un particolare frame. Il dizionario di collocazioni potrà inoltre estendere la sua trattazione a tutte quelle combinazioni (combinazioni libere e idiomatismi "parzialmente composizionali" che l'utente si attende ragionevolmente di trovare in un dizionario di tipo combinatorio. La risposta alla questione specifica se includere le unità polirematiche appare affermativa, visto che molte polirematiche (specie quelle con struttura $\mathrm{N}+\mathrm{A}$ ) sono tali da 
permettere all'utente di seguire proficuamente un percorso di ricerca secondo la direzione base $\nabla$ collocato. Anche la strutturazione semantica interna della voce deve risultare da un processo bottom-up, in modo che le accezioni rappresentino la «proiezione» delle collocazioni raccolte e trattate nel dizionario combinatorio. Ne conseguirà, rispetto ai dizionari dell'uso, una strutturazione della voce in parte diversa e, in alcuni casi, una maggiore granularità semantica.

Con un dizionario combinatorio rispondente ai criteri sopra descritti l'utente (professionale e non) della lingua potrà efficacemente completare il proprio corredo di opere linguistiche di consultazione, che tradizionalmente comprendono il dizionario generale e il dizionario dei sinonimi (e, in taluni casi, il dizionario analogico). La produzione linguistica attiva nella lingua madre, infatti, mette sovente nella necessità di reperire l'elemento lessicale che completi efficacemente il sintagma. Il dizionario sintagmatico (combinatorio) offre una efficiente modalità di ricerca della parola/del sintagma da abbinare, che sfrutta la base come punto di partenza, ossia come porta di accesso dall'elemento lessicale noto a quello non noto. 


\section{Riferimenti}

BISETTO, A. Composizione con elementi italiani. In GROSSMANN, M. \& RAINER, F. (Eds.). La formazione delle parole in italiano. Tübingen: Niemeyer, 2004, pp. 33-51.

DE MAURO, T. Grande Dizionario Italiano dell'Uso. Seconda edizione, Torino: UTET, 2007.

DE MAURO, T. Grande Dizionario Italiano dell'Uso. Versione elettronica, Prima edizione. Torino: Utet, 1999-2003.

DE MAURO, T. La fabbrica delle parole. Torino: Utet, 2005.

FESENMEYER, L. I recenti dizionari combinatori dell'italiano: un primo bilancio. In: Italienisch . Zeitschrift für italienische Sprache und Literatur 73/2015, pp. 100-131.

HÄCKI BUHOFER, A.; DRÄGER, M.; MEIER, S.; ROTH, T. Feste Verbindungen des Deutschen. Kollokationenwörterbuch für den Alltag. Tübingen: Francke Verlag, 2014.

HAUSMANN, F. J. Kollokationen im Deutschen Wörterbuch. Ein Beitrag zur Theorie des lexikographischen Beispiels. In: BERGENHOLTZ, H. \& MUGDAN, J. (Eds.). Lexikographie und Grammatik. Akten des Essener Kolloquiums zur Grammatik im Wörterbuch vom 28. bis 30.06.1984. Tübingen: Max Niemeyer, 1985, pp. 118-129.

JEŽEK, E. Lessico - Classi di parole, strutture, combinazioni. Seconda edizione, Bologna: Il Mulino, 2011.

LO CASCIO, V. Dizionario Combinatorio Compatto Italiano, John Benjamins, Amsterdam, 2012.

LO CASCIO, V. Dizionario Combinatorio Italiano, John Benjamins, Amsterdam, 2013.

MARELLO, C. Le parole dell'italiano: lessico e dizionari. Bologna: Zanichelli, 1996.

MASINI,F.Polirematiche, parole. In SIMONE R., BERRUTO G.D'ACHILLE P.(Eds.) Enciclopedia dell'italiano. Roma: Istituto della Enciclopedia italiana, 2011, pp. 1109-1112.

NEW OXFORD DICTIONARY OF ENGLISH, a cura di Judy Pearsall e Patrick Hanks. Oxford University Press: Oxford, 2001.

RUSSO, D. MdD. Modi di Dire. Lessico Italiano delle Collocazioni, Aracne, Roma, 2010.

SIMONE, R. Grande Dizionario Analogico della lingua italiana. Torino: Utet, 2010.

SIMONE, R. Nuovi fondamenti di linguistica. Milano: McGraw-Hill Education, 2013.

SIMONE, R. Verbi sintagmatici come costruzione e come categoria. In CINI, M. (Ed.), I verbi sintagmatici in italiano e nelle varietà dialettali. Stato dell'arte e prospettive di ricerca. Frankfurt am Main: Peter Lang, 2008, pp. 13-30.

SIMONE, R. Vocabolario Treccani - Il Conciso, Torino: Treccani, 1998.

TIBERII, P Dizionario delle collocazioni. Bologna: Zanichelli, 2012.

URZİ, F. Dizionario delle Combinazioni Lessicali. Convivium: Lussemburgo, 2009.

VOGHERA, M. Polirematiche, in GROSSMANN, M. \& RAINER, F. (Eds.), La formazione delle parole in italiano. Tübingen: Niemeyer, 2004, pp. 56-69.

ZINGARELLI, N. Lo Zingarelli 2016, a cura di Mario Cannella e Beata Lazzarini. Versione elettronica. Bologna: Zanichelli, 2015.

Recebido em: 03/11/2020

Aprovado em: 17/12/2020 